\title{
Le rôle de l'expérience dans la pratique de la géotechnique
}

\section{J.-P. MAGNAN}

Laboratoire central des ponts et chaussées

58, bd Lefebvre

75732 Paris Cedex 15
L'importance de l'expérience comme référence majeure pour les études géotechniques a été affirmée par tous les grands ingénieurs qui ont dû concevoir des projets ou analyser des accidents d'origine géotechnique. Cette conférence rappelle certaines de ces déclarations historiques puis analyse, pour les grandes catégories d'ouvrages géotechniques, les principes, données ou raisonnements qui sont issus de l'expérience et comment on pourrait les transmettre aux générations futures.

\section{The role of experience in geotechnical engineering practice}




\section{Introduction}

Les actes de congrès et de colloques, comme les revues de géotechnique et même la plupart des manuels d'enseignement donnent beaucoup d'informations sur le comportement des sols, les méthodes de calcul et les méthodes de mesure. Ils expliquent beaucoup plus rarement à quoi ressemblent les ouvrages géotechniques, quels problèmes attend l'ingénieur dans les études de conception de ces ouvrages, comment on les aborde et on les résout, en général dans un délai imposé et avec des moyens limités. En fait, Ils parlent peu de l'expérience, qui est l'autre pilier de la géotechnique et s'acquiert progressivement dans la vie professionnelle. C'est l'importance de l'expérience qui constitue le thème central dans cette conférence.

Dire que l'expérience joue un rôle majeur en géotechnique n'est pas une nouveauté. Si l'on cherche un peu dans des ouvrages publiés au $\mathrm{Xx}^{\mathrm{e}}$ siècle, on retrouve l'affirmation de l'importance de l'observation et de la prééminence de l'expérience. La section 2 de cette conférence regroupe quelques-uns de ces textes, dont il me semble que la méditation reste utile.

Dans les sections suivantes, la nature et la place de l'expérience sont analysées, tant au plan général que dans différents domaines de la géotechnique (problèmes d'ouvrages en terre, de fondations, de soutènements ou de pentes). On y voit que l'activité des géotechniciens s'appuie sur de nombreux outils de calcul, mais que la conception des ouvrages, l'évaluation des aléas et l'expertise des incidents ou accidents d'origine géotechnique sortent en partie du domaine de l'analyse déductive et mathématique pour utiliser des principes, des données et des raisonnements qui sont directement issus de l'expérience.

Nous verrons enfin comment la référence à l'expérience pourrait être formalisée dans l'enseignement de la géotechnique.

\section{2}

\section{Quelques extraits d'ourrages classiques}

Les citations regroupées ici ne traduisent pas de volonté d'exhaustivité. Les livres cités avaient pour eux d'être présents dans mes souvenirs ou dans ma bibliothèque. J'y ai retrouvé des idées qui, avec parfois quelques corrections d'époque, sont celles que je voudrais défendre ici.

Armand Mayer, dont je me souviens encore de la présence assidue aux réunions du Comité français de mécanique des sols dans les premières années de ma carrière, a publié en 1939 un petit (de format) ouvrage de 204 pages dans la collection "Section du Génie Civil n chez Armand Colin, sous le titre de Sols et fondations. L'avant-propos nous éclaire sur les pensées et objectifs de l'auteur:

«L'objet de ce petit ouvrage est d'indiquer au lecteur de quelle manière on peut aujourd'hui, en se plaçant au point de vue du constructeur, envisager les études de terrain et la préparation d'un projet de fondations. Nous avons cherché à montrer de quelle manière un certain nombre d'observateurs et de tech- niciens avaient essayé de combler le fossé qui séparait, jusqu'à très récemment, la géologie des travaux publics, en complétant la reconnaissance des terrains telle qu'elle résulte des études géologiques, par la mesure de leurs caractéristiques mécaniques, qui, seules, importent au constructeur. Après avoir indiqué la façon dont étaient exécutées les mesures, nous avons cherché à montrer comment on pouvait en utiliser les résultats. Nous nous sommes, à cet égard, soigneusement gardés de l'application des méthodes de l'analyse mathematique, dont le caractère rigoureux et imperturbable ne sert souvent qu'à dissimuler l'imprécision de l'hypothèse sur laquelle elle se fonde. Nous avons essayé, toutes les fois que nous l'avons pu, de partir des données de l'expérience pour en déduire plutôt l'allure des phénomènes que des conclusions générales précises, impossibles à formuler dans ce domaine. Nous avons d'ailleurs indiqué, à l'occasion de l'examen de quelques problèmes particuliers, l'écart considérable constaté entre les résultats expérimentaux et certaines déductions obtenues par le calcul et le plus généralement admises.

“La mécanique des sols n'en est pas encore à aspirer à la précision mathématique et tout notre effort à tendu à la vérification des hypothèses qui pourraient être prises dans l'avenir comme base d'une étude plus serrée. Dans certains cas, des résultats sont atteints. Dans d'autres, les essais sont en cours. D'autres fois enfin, l'hétérogénéité des sols fait craindre qu'il ne soit jamais possible de dépasser le stade de la connaissance qualitative. C'est d'ailleurs, il faut le reconnaitre, ce qui fait l'intérêt et le caractère passionnant de cette étude. Chaque problème est un cas particulier qui doit être étudié en soi-même, indépendamment de tous ceux qui ont pu être résolus antérieurement.

«L'application brutale d'une formule générale ne remplacera jamais l'observation directe et le raisonnement particulier; une solution ne vaudra que pour le cas qui aura été l'occasion de sa mise au point et toute extrapolation devra être précédée des vérifications indispensables. Ces observations, ces vérifications s'effectuent pour une part sur le terrain, pour une autre au laboratoire. Avant d'indiquer les méthodes que l'on y applique, qu'il nous soit permis de remercier ici tous ceux qui nous ont permis de réaliser le premier laboratoire français d'étude du sol et des fondations et en particulier, MM. Lasalle et Caloni, président et délégué général des Laboratoires du bâtiment et des travaux publics, ainsi que tous ceux, en particulier M. Charles Granger, qui y ont collaboré avec nous depuis sa fondation et l'ont amené à son degré de développement actuel. y)

En 1949, Caquot et Kerisel publient un Traité de mécanique des sols, présentẻ comme la deuxième édition du livre antérieur d'A. Caquot intitulé Équilibre des massifs à frottement interne. Stabilité des terres pulvérulentes et cohérentes (1934), La lecture de la préface ne donne pas la mème impression que celle du texte de Mayer. J'ai gardé tout de même des extraits de cette préface, car elle me semble typique de l'autre regard sur la géotechnique, considérée comme une branche de la mécanique.

«La mécanique des sols concerne l'application des lois de la mécanique et de l'hydraulique aux problèmes qui se posent à propos de l'étude des sols de fondation: elle constitue donc un chapitre de la résistance des 
matériaux, spécialement applicable aux matériaux constitutifs de l'écorce terrestre que l'on trouve dans les zones où l'Ingénieur et l'Architecte sont amenés à construire des ouvrages de génie civil ou des bâtiments. [...]

"Les problèmes de ce genre se sont posés à I'homme depuis la plus haute antiquité, aussi bien à l'homme primitif pour fonder sur pilotis ses premières cités lacustres qu'aux bâtisseurs des cathédrales.

«Au XVII siècle, la mécanique des sols est étudiée pour la première fois et la plus importante contribution fut apportée par la France et en particulier par les ingénieurs militaires du Roi; plus de la moitié des traités publiés dans le monde sur la matière avant 1850 émanent de Français. L'essai de Coulomb de 1773, présenté à l'Académie royale des sciences à Paris [...] est le traité fondamental, suivi par de nombreuses études énonçant pour la première fois la loi de base correcte, qui va déterminer toutes les méthodes scientifiques d'investigation.

«Dans les dernières années, cette science s'est surtout développée grâce aux recherches dans les pays où la médiocrité du terrain de fondation posait des problèmes essentiels à l'activité des Ingénieurs et Architectes. C'est ce qui explique la contribution importante apportée par les écoles hollandaise et viennoise. Qu'il s'agisse des sols alluvionnaires des Pays-Bas ou de la vallée du Danube, les problèmes difficiles de fondations dans ces deux régions appellent des études scientifiques pour les édifices à construire. [...]

«Lorsque l'on construit un pont, par exemple, on travaille en utilisant des matériaux manufacturés et homogènes dont les propriétés mécaniques sont bien connues et néanmoins l'on admet dans le calcul des coefficients de sécurité qui ne sont jamais inférieurs à 2 . Il n'en est pas de même dans la mécanique des sols: les formations géologiques et les propriétés des sols de fondation varient d'un point à l'autre et sont souvent complexes. En raison de ces circonstances, les coefficients de sécurité réels étaient autrefois souvent bien inférieurs aux précédents, comme dans d'autres cas beaucoup plus élevés, La science du sol permet aujourd'hui de sortir de cette incertitude.

«Certains ont voulu apercevoir dans la mécanique des sols deux écoles, l'une théorique, l'autre expérimentale. Il n'en est plus de même aujourd'hui où l'expérience fournit à la théorie les bases correctes, les déductions mathématiques qui en résultent étant ensuite vérifiées par l'expérience.

«Les noms des Français Coulomb, Poincelet, Poisson, Darcy, Boussinesq et Résal dominent les études théoriques de la mécanique des sols; mais ainsi que le note un savant anglais, M. Golder, qui vient de faire une étude comparative des laboratoires des sols dans les différentes nations européennes, les études expérimentales dans notre pays n'ont pas suivi le même rythme.

«Les laboratoires en service aujourd'hui comblent cette lacune. [...]

«Rompant délibérément avec les méthodes semiempiriques usuelles de calcul des tassements qui consistent à rechercher d'abord les composantes verticales des contraintes agissantes, nous les avons calculées d'après l'intégralité du tenseur par la formule de Boussinesq. [...]

«Nous montrons d'ailleurs que tout phénomène de mécanique des sols exige l'examen séparé des deux tenseurs solide et liquide: la courbe intrinsèque qui apparaît dans un essai rapide ne correspond à aucune réalité physique. [...]»)

C'est un bel exemple de confiance dans l'approche française des siècles précédents, où le comportement de la nature doit par principe découler de quelques lois de base, déjà connues à l'époque.

Les réflexions de $\mathrm{K}$. Terzaghi considèrent la géotechnique d'un point de vue qui est certainement plus proche de celui d'A. Mayer que de celui de Caquot et Kerisel. J'ai retenu quelques courts passages du livre intitule From theory to practice in soil mechanics. Selections from the writings of Karl Terzaghi (préparé par L. Bjerrum et al. par publié en 1960 par John Wiley and Sons, trois ans avant son décès) et d'un article récent de R.E. Goodman intitulé " Karl T'erzaghi's legacy in Geotechnical Engineering p et publié dans le numéro d'octobre 2002 du magazine Geo-Strata de l'ASCE. R.E. Goodman est, par ailleurs, Y'auteur d'un livre intitulé Karl Terzaghi, the engineer as artist.

\section{R.E.Goodman écrit :}

«Karl Terzaghi (1883-1963) fut le premier à élaborer une mécanique des sols complète, avec la publication de Erdbaumechanik en 1925. Sa formulation du principe des contraintes effectives et de son influence sur le calcul des tassements, la résistance, la perméabilité et l'érosion des sols sont sa contribution la plus prodigieuse. Mais Terzaghi a aussi eu un rôle de pionnier pour un grand nombre de méthodes et de procédures de reconnaissance, d'analyse, d'essai, d'instrumentation et de pratiques qui définissent le champ que nous appelons maintenant géotechnique.

« Parmi les publications, rapports et conférences de Terzaghi, on trouve des contributions stimulantes sur un domaine très large, incluant: les méthodes de classification des sols et des roches, les phénomènes de capillarité dans les sols, la théorie et l'observation de la consolidation et du tassement, l'érosion interne et sa prévention, la conception et la construction des barrages en terre, en enrochements et en béton sur tous types de fondations, les ancrages dans le sol pour les ponts suspendus, la mesure en place et en laboratoire des pressions interstitielles et des propriétés des sols, l'utilisation des réseaux d'écoulement en deux et trois dimensions, la conception des puits de drainage et des tunnels, la conception des ouvrages fluviaux et maritimes pour résister à l'érosion, les variations de la pression des terres sur les murs et les rideaux, la construction dans les zones de pergélisol, les fondations sur pieux, l'amélioration des sols par compactage, par battage de pieux, par injection et incorporation de géotextiles, les tunnels dans les sols et les roches, la géologie de l'ingénieur, la formation et l'effondrement des entonnoirs karstiques, les affaissements régionaux dus aux pompages pétroliers et les glissements de terrain. [...]

"Bien qu'il ait été un grand éducateur, Terzaghi développa progressivement une suspicion envers l'éducation formelle, dont il pensait qu'elle pouvait obscurcir l'observation des nouveaux phénomènes. Il exprimait la plus grande admiration pour les autodidactes qui apprenaient en ouvrant leurs yeux et leurs esprits. [...]

"Lorsque l'on passe en revue l'étendue de ses contributions et sa maîtrise du domaine, il est intéressant d'examiner la formation et les intérêts de Terzaghi, 
ainsi que sa philosophie et ses méthodes de travail. Son éducation combinait la rigueur et la formation militaire autrichiennes avec une passion pour l'observation des sciences naturelles et la contemplation de la beauté de la nature. Ses intérêts étaient très variés: la construction, la géologie, les mathématiques, la philosophie et l'éthique, l'architecture, les fleurs, la natation, la conversation, les voyages, la littérature, la musique, l'art, les femmes, les hommes et l'écriture. [...]

«Les objectifs de Terzaghi [...] changèrent brutalement vers l'âge de 43 ans (1926). Pendant sa vie d'homme jeune, il avait cherché à développer une méthodologie d'analyse rationnelle, analytique ou empirique, qui tienne compte des contraintes géologiques, pour le calcul des ouvrages fondés sur les sols (et, dans une moindre mesure, les roches). A l'âge mūr, ayant atteint ce premier objectif, il poursuivit avec passion sa pratique de l'ingénierie pour tester et tempérer les méthodes émergentes par les réalités physiques. Dans cette démarche, il fut de plus en plus préoccupé par la difficulté d'en savoir assez sur la morphologie et les propriétés du site pour fixer la conception du projet avant le début de la construction. Cette préoccupation l'entraîna encore plus vers l'observation de la réponse du sol et de l'ouvrage pendant la construction afin d'alimenter une révision permanente des projets, ce qui fit de lui, avec son collaborateur Ralph Peck, un partisan et un praticien de la "méthode observationnelle". Malgré les contributions considérables de Terzaghi aux progrès de la théorie de la mécanique des sols, il conseillait pour cette raison avec insistance à la profession de rester en contact avec le comportement des sols réels dans la pratique de la géotechnique. [...]

«Karl Terzaghi fut un ingénieur remarquable et passionné. Comme il le dit lui-même: "Toutes les modestes réalisations que j'ai à mon crédit peuvent être décrites par une formule simple... Guidé par le bon sens et des observations fortuites, j'ai reconnu les points faibles des procédures traditionnelles et j'ai essayé de les rendre moins faibles. Parfois j'ai échoué mais en général j'ai réussi". $n$

Dans les cuvres écrites de Terzaghi, les discours d'ouverture des Congrès de mécanique des sols de Cambridge (États-Unis), en 1936, et de Londres, en 1957, illustrent les remarques précédentes. En 1936, K. Terzaghi termine son discours par ces phrases:

« [...] la fonction de cette conférence est simple. Elle consiste essentiellement à établir des contacts personnels entre ceux qui sont intéressés par la mécanique des sols du point de vue de la théorie ou de la pratique et de stimuler les échanges d'expérience. Bien qu'elle soit apparue il y a moins de 25 ans, la mécanique des sols est déjà assez vieille pour avoir acquis la modestie qui naît de l'expérience. Nous savons aujourd'hui que rien d'utile ne peut être accompli dans cette discipline sans la coopération intelligente et patiente de l'ingénieur praticien sur le terrain. Certaines des contributions les plus importantes à ce congrès sont le résultat direct d'une telle coopération. Pour cette raison, nous sommes très heureux d'accueillir parmi les participants du congrès un grand nombre de dirigeants remarquables et d'ingénieurs expérimentés du monde de la construction. Comme ces hommes doivent leur succès et leur position professionnelle à leur capacité de distinguer la réalité de la fiction, je suis sûr qu'ils vont apprécier nos sentiments envers la sagesse des manuels à demi cuits et nous aider à descendre vers les faits tangibles. $)$

\section{Dans son allocution de 1957, il déclare:}

«[...] Cette conclusion me conduit au dernier sujet de mon discours, qui est la façon de transmettre efficacement ces connaissances et ces visions nouvelles à la génération suivante. Pour répondre à cette question, nous devons examiner les pré-requis des activités artistiques au sens le plus large du terme.

$\propto$ Pour pratiquer un art avec succès, il faut posséder la capacité, attribuée à Théodore Roosevelt, de "penser avec ses hanches". En d'autres termes, il faut être capable d'arriver à des conclusions correctes sans passer par un raisonnement logique. [...]

«Le même processus de développement peut être retrouvé dans la vie de quasiment tout ingénieur qui a acquis une réputation justifiée de ajugement sain ». Lorsqu'il était étudiant, il travaillait avec sa tête en allant aussi loin que le raisonnement logique pouvait l'emmener. Au début de sa vie professionnelle, la partie utile de ce qu'il avait appris est progressivement entrée dans son subconscient et il a pu alors aborder sans grand risque des problèmes d'ingénierie qui ne pouvaient être résolus par des procédures rationnelles, ce qu'il ne pouvait faire plus tôt. Les étudiants qui observent un tel ingénieur en action peuvent en déduire que le temps qu'ils doivent passer à absorber de la théorie est du temps perdu, mais cette conclusion serait erronée. L'ingénieur qu'ils observent n'aurait jamais atteint un tel niveau de perfection s'il n'avait d'abord appris et complètement digéré chacun des nodules d'information qui ont une utilité directe pour ses activités professionnelles. Les détails des théories peuvent sortir de sa mémoire, mais leur essence devient de plus en plus active au fil des ans. Pour cette raison, le secret d'une éducation professionnelle efficace réside dans l'affirmation permanente de ce qui mérite d'être absorbé et ensuite digéré, et sur les incertitudes qui sont inévitablement associées à l'application des théories mathématiques aux problèmes d'ingéniérie. »)

Je ne saurais enfin assez recommander à tous les ingénieurs, et notamment à ceux qui consacrent leur vie à la géotechnique, de lire l'ouvrage préparé par J. Dunnicliff et D.U. Deere et publié en 1984 par John Wiley and Sons sous le titre Judgement in geotechnical engineering. The professional legacy of Ralph B. Peck.

La question de la place de l'expérience parmi les outils des ingénieurs géotechniciens est donc débattue depuis longtemps, mais elle reste d'actualité.

3

\section{Qu'est-ce que l'expérience}

L'expérience peut être définie de différentes façons: elle peut recouvrir les capacités qu'acquiert l'ingénieur dans sa vie professionnelle, à la fois par l'observation, l'analyse de problèmes variés et la réflexion, ou ce qu'il a appris après la fin de sa formation. Cette expérience est implicitement une expérience individuelle.

Mais on peut aussi définir l'expérience «en creux» par rapport à une idée citée plus haut, qui est que l'approche scientifique utilise les mathématiques pour 
déduire de lois physiques simples (et déjà connues) les comportements des massifs de sols que l'on valide par d'autres études expérimentales. L'expérience est alors tout ce qui, dans l'activité des ingénieurs, ne répond pas à cette définition. Il ne faut pas en déduire que l'expérience est dans ce cas tout ce qui est «non scientifique m: elle est aussi scientifique que la construction de modèles, mais selon une autre logique.

En géotechnique, la liste des connaissances et des outils issus de l'expérience est longue. Elle comprend à la fois les règles de gestion de la sécurité des ouvrages, l'appréciation des écarts entre les résultats de calculs et la réalité, certaines méthodes de calcul, les règles pratiques de programmation et d'interprétation des reconnaissances géotechniques, les dimensions courantes ou optimales des ouvrages, la connaissance des aléas et l'évaluation des risques. L'expérience est alors à la fois individuelle et collective et résulte en grande partie de l'activité de grands laboratoires, centres de recherche ou bureaux d'ingénierie comme ont pu l'être, en France, les services techniques d'EDF, le Centre expérimental du bâtiment et des travaux publics (CEBTP) et les services techniques du ministère des Travaux publics, puis de l'Équipement: Service technique des routes et autoroutes (SETRA), conjointement avec le Laboratoire central des ponts et chaussées ( $\mathrm{LCPC}$ ) et le réseau des laboratoires régionaux des ponts et chaussées puis des centres d'études techniques de l'équipement (CETE), Centre d'étude des tunnels (CETU), Centre d'études techniques maritimes et fluviales (CETMEF). Beaucoup de pays ont créé des organismes aux fonctions comparables, TRRL et BRE au Royaume-Uni, BASt et BAW en Allemagne, CEDEX en Espagne, LNEC au Portugal, les instituts géotechniques suédois, norvégien et danois, GeoDelft aux Pays-Bas, le Laboratoire géotechnique de l'État, le CRR et le CSTC en Belgique... La liste est longue, mais traduit assez bien l'importance accordée à la maîtrise des connaissances et techniques de la géotechnique par l'action collective, associant la pratique de la géotechnique et la recherche appliquée, souvent avec des outils de calcul mais toujours avec des démarches appuyées sur l'expérimentation et l'observation de la nature et des ouvrages.

\section{4}

\section{Exemples}

\section{1}

\section{Généralités}

Le rôle majeur de l'expérience dans la pratique de la géotechnique est lié aux limites de la modélisation du comportement des sols, des roches et des ouvrages.

La démarche de conception des ouvrages manufacturés du génie civil et du bâtiment s'est structurée autour de l'étude séparée de la fabrication et des propriétés et des matériaux, d'une part, et du calcul, d'autre part, l'incertitude provenant pour l'essentiel des charges: le modèle géométrique est fixé d'abord; le comportement des matériaux est connu et l'on peut spécifier les valeurs des paramètres; le modèle de calcul fait le reste, avec quelques «garde-fous $n$ issus de la pratique (règles de ferraillage, de soudure, etc.).

La géotechnique s'est développée dans un autre univers: la nature des matériaux n'est pas connue a priori et ils peuvent avoir des propriétés très différentes, il existe de nombreux modèles de calcul, mais qui sont parfois adaptês à un seul type d'ouvrage et parfois à un seul type de sol; les techniques de construction peuvent modifier sensiblement l'interaction des sols et des ouvrages. Si les procédures de conception des structures et des fondations pouvaient être jugés aussi empiriques au cours du XIX ${ }^{e}$ siècle, le passage au calcul mécanique est intervenu beaucoup plus vite pour les structures que pour les fondations. Terzaghi (1951) rapporte qu'à la fin du XIX ${ }^{e}$ siècle la conception des fondations s'appuyait sur une étude sommaire d'identification des matériaux et qu'on associait à chaque type de matériau une charge admissible, sans considération pour les tassements. Il fallut de nombreux accidents pour que les ingénieurs se rendent compte que d'autres facteurs entraient en compte, et notamment la déformabilité, donc l'état des sols.

Les développements de la géotechnique ont été faits pour l'essentiel à l'occasion d'études de grands ouvrages ou d'expertises de catastrophes, avec quelques périodes de restructuration des connaissances, comme celle de Terzaghi dans les années 1920, le développement de la mécanique des roches dans les années 1950 ou de la mécanique des sols non saturés dans les années 1980. Ils ont en général produit d'abord des méthodes de prévision simplifiées, calées sur l'expérience, avec des paramètres issus de méthodes d'essai rustiques, qui ont été perfectionnées par la suite. Les perfectionnements des essais, comme des méthodes de calcul ont été faits dans les laboratoires ou centres de recherche, parallèlement aux activités d'études et d'expertise des ingénieurs. Ils ont parfois duré de lonques années et se sont spécialisés sur des objectifs autonomes, sur lesquels on a accumulé beaucoup d'outils et de connaissances, alors que les ingénieurs pouvaient avoir d'autres besoins, qu'ils ont cherché à résoudre d'autres façons.

Par exemple, on a consacré beaucoup de travail à l'étude en laboratoire des propriétés mécaniques des argiles reconstituées et des sables purs et au développement de théories puis de modèles numériques pour représenter leurs déformations et leur rupture. Mais les ingénieurs devaient répondre pendant ce temps à des questions sur la stabilité et les déformations de sols réels qui sont rarement des argiles ou des sables purs et que l'on a souvent du mal à prélever pour les apporter dans les laboratoires d'essais. Les essais en place, dont aucun n'a de théorie pour rattacher ses résultats au comportement des ouvrages, sont un exemple des outils dont l'utilisation est avant tout justifiée par l'expérience et le calage de formules de calcul sur le comportement observé d'ouvrages en vraie grandeur ou de modèles réduits. Beaucoup de règles de conception ou de calcul de la géotechnique sont issues d'une telle démarche. Elles constituent l'essentiel de l'expérience collective de la profession géotechnique.

L'expérience individuelle différencie pour sa part la capacité des ingénieurs à a inventer $»$ le modèle géotechnique du site à partir de l'information disponible, à détecter rapidement les facteurs explicatifs d'un accident, à deviner les dimensions optimales d'un ouvrage ou les incertitudes qui peuvent rendre un projet risqué.

Nous évoquerons ces deux types d'expérience dans les exemples qui suivent. 


\section{L'expérience et le calcul des fondations}

Le calcul moderne des fondations profondes repose dans la majorité des pays sur les résultats d'essais en place (pénétromètre statique ou dynamique, SPT, pressiomètre), qui sont reliés au frottement latéral et à la résistance de pointe des pieux par des relations empiriques, tenant compte de la nature des sols et du mode d'exécution des pieux. La modélisation numérique a une place dans les calculs d'interaction avec d'autres structures. Le calcul des moments de flexion dans les pieux utilise en général un modèle de poutre sur appui continu ou des distributions forfaitaires de pressions de contact. Toutes ces procédures comportent des parties empiriques qui appartiennent à l'expérience collective d'un organisme ou d'un pays.

Le calcul des fondations superficielles est dans une situation différente, puisqu'il existe des solutions théoriques fondées sur l'analyse des équilibres limites ou sur l'analyse limite (qui tient compte des conditions imposées aux déformations et pas seulement de l'équilibre des contraintes), et utilisant les paramètres c et $\varphi$ introduits du temps de Coulomb (XVII' siècle). Le calcul des fondations superficielles à partir des essais en place est aussi pratiqué.

Les limitations des méthodes d'analyse mathématiques ou numériques sont liées d'une part à la variabilité des sols naturels et de leurs propriétés physiques et mécaniques, et d'autre part à l'absence de la déformabilité des sols dans l'analyse de stabilité (à part le caractère associé de la loi d'écoulement plastique, qui ne correspond que rarement aux données expérimentales).

Les calculs de tassements s'effectuent soit par des méthodes simplifiées utilisant les composantes verticales des contraintes, soit par des calculs en éléments finis.

Les fondations profondes s'appuient donc plutôt sur l'expérience collective et les fondations superficielles sur des procéciures d'analyse mécanique reliées à la mécanique des milieux continus, renvoyant sur le choix des paramètres, c'est-à-dire le modèle géotechnique du site avec les incertitudes que l'expérience personnelle aide à apprécier.

\section{3}

\section{L'expérience et les études de pentes naturelles}

Les études de stabilité de pentes s'effectuent en pratique par analyse de mécanismes de glissement potentiel sur des surfaces de rupture. Les calculs se font par des méthodes numériques suivant des procédures variées. Le principal problème est de déterminer les propriétés de résistance du sol. Pour les massifs homogènes et les glissements de faibles dimensions. quelques essais de laboratoire peuvent fournir des données fiables. Mais le problème reste complexe pour les grands glissements de versants, dont les terrains (sols et roches) échappent parfois à toute mesure directe. Lorsqu'un glissement s'est déjà produit, les paramètres peuvent être estimés par une analyse des conditions d'équilibre de la masse instable. La progressivité des mouvements vient compliquer cette analyse. L'évolu- tion des glissements au cours du temps échappe généralement au calcul et les méthodes de prévision s'appuient souvent sur des corrélations avec des mesures hydrauliques (précipitations, niveau des eaux souterraines...). Globalement, le domaine des études de pentes comporte donc d'une part un champ classique où l'on peut utiliser des outils validés par l'expérience collective et d'autre part un champ moins bien connu où l'expérience individuelle a un plus grand rôle.

\section{4}

\section{L'expérience et les travaux de terrassements}

Les études de terrassements cherchent à prévoir l'emploi dans les remblais des matériaux extraits des déblais en cours de chantier. La variabilité des terrains, que l'on ne peut complètement caractériser pendant les reconnaissances géotechniques, gêne la prévision des mouvements de terres pendant la période de préparation des projets. Les études de compactage s'appuient le plus souvent sur des essais de compactibilité de type Proctor, que l'on effectue sur les différents types de matériaux rencontrés. La cadence des grands chantiers conduit à privilégier des spécifications d'utilisation des matériaux en remblais qui fixent les conditions de compactage en fonction de la nature et de l'humidité des terrains extraits des déblais. Ces spécifications sont du domaine de l'expérience collective. Mais la définition des terrains que l'on trouvera dans un déblai dépend beaucoup plus de l'expérience personnelle du géotechnicien chargé de l'étude.

\section{5}

\section{L'expérience et les constructions sur sols compressibles}

Une réflexion détaillée sur l'apport de l'expérience dans ce domaine de la géotechnique a été présentée par l'auteur au $13^{\circ}$ Congrès régional africain de mécanique et de géotechnique (Magnan, 2003c). Du point de vue de la "démarche scientifique» de résolution des problèmes de géotechnique, avec des modèles de calcul construits sur des lois physiques reconnues, le calcul du comportement des massifs de sols argileux pourrait constituer, comme l'analyse de la stabilité des fondations superficielles, un champ idéal. On dispose de programmes de calculs tridimensionnels en éléments finis, les sols peuvent être prélevés et transportés jusqu'aux laboratoires pour y subir des essais œedométriques et triaxiaux. De nombreuses expérimentations ont été réalisées pour valider les modèles et on peut considérer que l'on dispose d'outils pour faire les projets. Mais la pratique montre que cela ne suffit pas encore: des ruptures de remblais viennent rappeler périodiquement qu'il faut rester prudent et il est fréquent que les prévisions de tassements soient fausses. Cela montre déjà que l'utilisation de ces outils nécessite une certaine expertise de la part des ingénieurs responsables des projets et des travaux. Mais quand on analyse de plus près les outils de ces ingénieurs, on découvre que beaucoup d'outils sont issus d'une démarche d'observation directe de la nature et ne sont pas réductibles aux méthodes de calcul issues de la mécanique des milieux continus. On peut citer par exemple la relation de proportionnalité des déplace- 
ments latéraux et des tassements sous les remblais ou les ordres de grandeur des parties des déformations associées à la compressibilité cedométrique et au fluage, ou bien encore le fait que la stabilité à court terme implique la stabilité à long terme du même remblai.

De façon plus générale, cet exemple comme les précédents suggère qu'il y a dans la pratique de la géotechnique le niveau des outils de prévision, qui repose sur des analyses mécaniques ou sur l'expérience collective, et le niveau de l'élaboration du modèle géotechnique, où l'expérience collective prépare des outils mais l'expérience personnelle des ingénieurs reste un facteur essentiel. Si l'on cherche à comprendre quelle fut la particularité des grands ingénieurs qui ont marqué la géotechnique, c'est probablement dans leur capacité à comprendre la nature et son interaction avec le fonctionnement des ouvrages et à trouver des modèles d'analyse adaptés qu'on pourra trouver la réponse.

\section{6}

\section{L'expérience et les travaux de soutènement}

Les études de soutènements ont des caractéristiques proches des études de fondations. Les théories de la poussée et de la butée sont parentes des théories de la portance des sols et elles ont les mêmes possibilités et les mêmes limitations: des outils connus à base théorique, peu d'intérêt pour les déformations, une interaction forte avec le calcul des structures qui a fait utiliser des modèles d'interaction locale (de type coefficient de réaction). La détermination des paramètres de résistance des sols est parfois complexe, notamment quand les matériaux en contact avec le soutènement sont des remblais compactés. L'expérience garde un rôle important dans le choix des modèles géotechniques et dans la conception générale des ouvrages.

\section{5}

\section{Enseigner ou transmettre l'expérience}

La transmission des outils de la géotechnique d'une génération à la suivante est une préoccupation importante pour la profession géotechnique dans son ensemble mais aussi pour ses clients. La formation des géotechniciens ne se limite pas à l'enseignement de la représentation des sols et des roches et des méthodes de calcul. Il me parait tout d'abord important de combiner l'apprentissage conjoint de la mécanique des sols et de la mécanique des roches, selon les vœux exprimés par Pierre Habib dans le discours de clôture du Symposium international d'Athènes sur les sols indurés et les roches tendres en 1997. De la même façon, les géotechniciens me paraissent devoir connaître les modes de fonctionnement et les règles générales de la conception des structures en béton armé et en acier, ou les lois générales de l'hydraulique à surface libre. On peut enseigner aussi les conclusions des études expérimentales et des observations qui aboutissent à la formation de l'expertise que j'ai qualifiée plus haut de collective. Mais ce ne sont que les préambules de la transmission de l'expérience.

Pour sa part, la transmission de l'expérience individuelle pourrait utilement bénéficier de livres qui rassembleraient des descriptions de projets, avec les modes de raisonnement de géotechniciens spécialistes, ou donneraient des recommandations pour la conception des ouvrages, la définition des modèles géotechniques, ou la façon d'aborder les expertises. Ce genre de littérature est pratiquement absent de nos librairies.

Mais la transmission de l'expérience individuelle passe tout de même d'abord par une relation personnelle de type «maitre à élève» entre un géotechnicien expérimenté et un géotechnicien en dẻbut de carrière. Une relation qui s'établisse autour de projets réels, de plus en plus complexes. Peut-on organiser cette transmission dans les établissements d'enseignement supérieur? Faut-il attendre le début de la vie professionnelle et l'organiser dans un bureau d'étude ou une entreprise, ou dans un cadre professionnel collectif? Ces choix dépendent de beaucoup de facteurs et doivent être adaptés aux conditions locales. Mais il faut que cette transmission d'expérience soit organisée.

\section{6}

\section{Conclusion}

Cette réflexion sur le rôle de l'expérience dans la pratique de la géotechnique complète des travaux récents sur la nature de l'activité des géotechniciens ("L'organisation du travail en géotechnique: développement, normalisation et artisanat », Magnan, 2001), sur la place de la géotechnique dans l'organisation des projets et des travaux (Magnan, 2003a) et sur la place des normes dans la pratique de la géotechnique (Magnan, 2003b). La profession géotechnique se trouve, comme sans doute à d'autres occasions dans son histoire, à un carrefour où elle doit intégrer les influences du développement des ordinateurs et des méthodes de calcul numérique, les effets directs et indirects de la tendance de la société à généraliser à toutes les activités une approche normative qui s'accommode mal du besoin d'expérience et de compétence artisanale dans toutes les activités qui touchent à la nature, de la place croissance de recherches qui veulent développer la part déductive de la mécanique des sols et des roches aux dépens des domaines régis par l'expérience, mais souvent sans connaitre l'importance réelle de l'expérience et la valeur des modes de connaissance non déductifs pour la gestion des systèmes complexes.

Je suis convaincu que la géotechnique ne peut être pratiquée que sur un mode artisanal, pour étudier des ouvrages qui restent uniques. La transmission de l'expérience collective et individuelle en est la condition, 
Bjerrum L, Casagrande A., Peck R.B., Skempton A.W. (1960) - From theory to practice in soil mechanics. Selection from the writings of Karl Terzaghi. John Wiley and Sons, New York, London, $425 \mathrm{p}$.

Caquot A. (1934) - Equilibre des massifs à frottement interne. Stabilité des terres pulvérulentes et cohérentes. GauthierVillars, Paris.

Caquot A., Kerisel J. (1949) - Traité de mécanique des sols. Gauthier-Villars, Paris, $385 \mathrm{p}$.

Dunnicliff J., Deere D.U. (1984) - Judgement in geotechnical engineering. The professional legacy of Ralph B. Peck. John Wiley and Sons, New York, 332 p.

Goodman R.E. (1999) - Karl Terzaghi, the engineer as artist. ASCE Press, Reston, VA, 340 p.

Goodman R.E. (2002) - Karl Terzaghi's legacy in geotechnical engineering. Geo-Strata, ASCE, October.
Magnan J.-P. (2001) - L'organisation du travail en géotechnique; développement, normalisation et artisanat. Conférence en l'honneur des 21 ans du CERMES. Ecole Nationale des Ponts et Chaussées, Marne-la-Vallée, 21 septembre 2001. Revue Française de Géotechnique, $n^{\circ} 99,2001$, p. 73-80. Publié dans « La lettre de la géotechnique », $n^{\text {os }} 26$ et 27 mars et juin 2002, Société internationale de mécanique des sols et de géotechnique,

Magnan J.-P. (2003a) - La place de la géotechnique dans l'organisation des projets et des travaux, Conférence à la réunion de l'ALBTP et du CTGA, Yacundé, 29 mars 2003. Publié dans « La lettre de la géotechnique $n, n^{\circ} 31$ et 33 . juin et décembre 2003, Société internationale de mécanique des sols et de géo. technique,
Magnan J.-P. (2003b) - Les normes et la pratique de la géotechnique. Conférence à la réunion de l'ALBTP et du CTGA, Yaoundé, 29 mars 2003. Publié dans « La lettre de la géotechnique », n"32, septembre 2003, Société internationale de mécanique des sols et de géotechnique.

Magnan J.-P. (2003c) - Quelques leçons de la pratique des projets et des travaux de construction dans les zones de sols compressibles, $13^{\circ}$ congrès régional africain de mécanique des sols et de géotechnique, Marrakech, 8 décembre 2003.

Mayer A. (1939)-Sols et fondations, Armand Colin, Paris, $203 \mathrm{p}$.

Terzaghi K. (1951) - The influence of modern soil studies on design and construction of foundations. Building Research Congress, Div. 1, Part III, p. 139-145. 